\title{
Acknowledgements of AICAT2016
}

CC Akadémiai Kiadó, Budapest, Hungary 2017

The Guest Editors are very grateful to the following scientists for their critical and valuable reviews which have significantly contributed to the quality of the papers in this special chapter.

Bikiaris, D., Greece

Biondi, M., Italy

Braissant, O., Switzerland

Casanova, C., Spain

Castronuovo, G., Italy

Cavallaro, G., Italy

Coquerel, G., France

da Costa Monteiro, E. E., Brazil

Fessas, D., Italy

Flandorfer, H., Austria

Iaccarino, N., Italy

Karolewicz, B., Poland

Kozbial, M., Poland

Krutova, O., Russian Federation

Lazzara, G., Italy

Ledeti, I. V., Romania

Marongiu, B., Italy

Monajjemzadeh, F., Iran

Muntean, C., Romania

Mustata, F., Romania

Neves Jr., A., Brazil

Ortiz de Zárate, J. M., Spain

Petraccone, L., Italy

Pielichowski, K., Poland

\author{
Popescu, C., Germany \\ Prnová, A., Slovakia \\ Ribeiro da Silva, M. D. M. C., Portugal \\ Sbirrazzuoli, N., Italy \\ Scampicchio, M., Italy \\ Schiraldi, A., Italy \\ Sgarlata, C., Italy \\ Siciliano, C., Italy \\ Stefanescu, M., Romania \\ Suñol Martinez, J. J., Spain \\ Tabero, P., Poland \\ Terekhova, I. V., Russian Federation \\ Tiné, M. R., Italy \\ Trník, A., Slovakia \\ Usacheva, T. R., Russian Federation \\ Valenzuela Diaz, F. R., Brazil \\ Vecchio Ciprioti, S., Italy \\ Vittadini, E. G., Italy \\ Vlase, G., Romania \\ Vlase, T., Romania \\ Williams, P. J., South Africa \\ Wyrzykowski, D., Poland \\ Zelic, J., Croatia
}

